fait il y a très peu de néo-platonisme en jeu dans le dialogue des trois amis. Parmi les autres lecteurs contemporains du Premier Curieux figurent Simon Goulart et Pantaléon Thevenin, tous deux commentateurs de La Sepmaine de du Bartas, aussi bien que Claude Duret. Aujourd'hui, ce sont de nouveaux lecteurs qui profiteront d'une aide très précieuse grâce à l'érudition vaste et juste de Jean Céard.

STEPHEN MURPhy, Wake Forest University

\title{
Pontus de Tyard.
}

Erreurs amoureuses (1549), texte établi et annoté par Guillaume de Sauza. Saint-Étienne: Publications de l'Université de Saint-Étienne, 2009. 165 p. 9782-86272-513-0 (broché) $9 €$

Dans la collection « Textes et Contre-textes » des Publications de l'Université de Saint-Étienne, Guillaume de Sauza met à notre disposition le texte des Erreurs amoureuses établi d'après l'édition Jean de Tournes de 1549. Est donc édité ici le seul premier livre : rappelons qu'il sera suivi en 1551 d'une Continuation puis, en 1555, d'une tierce partie. Cette édition se démarque donc des trois autres grandes éditions modernes des Erreurs, qui donnent toutes le texte des trois livres, et avec lesquelles elle ne fera pas double emploi : celle de John C. Lapp (Paris, STFM-Marcel Didier, 1966), qui donne les Euvres poétiques complètes en se fondant sur le texte de 1573 (Les Euvres poetiques de Pontus de Tyard..., Paris, Galiot du Pré), celle de John A. McClelland (Genève-Paris, Droz-Minard, 1967), qui se fonde sur le texte de 1555, enfin celle d'Eva Kushner, qui constitue le premier tome des Euvres complètes (Paris, Champion, 2004) et se fonde aussi sur l'édition de 1573.

Cette édition s'ouvre sur une introduction sobre d'une vingtaine de pages. Après un bref résumé du parcours de Pontus de Tyard, G. de Sauza rappelle l'importance des Erreurs de 1549 dans le cadre de l'émergence du pétrarquisme français : spécificité du recueil par rapport à L'Olive de Du Bellay, ambiguïté du titre qui remotive un lieu commun de la rhétorique pétrarquiste, combinant les idées d'errance spatiale, d'erreur intellectuelle, de " cheminement tortueux du sujet lyrique » (p. 7). L'introduction fait également le point sur les sources 
littéraires, privilégiant l'importance prépondérante du modèle scévien, auquel s'ajoutent le pétrarquisme italien, la poésie courtoise, et la métaphysique néoplatonicienne, essentiellement représentée par Ficin et Léon Hébreu, dont Tyard traduit les Dialogues d'amour alors même qu'il rédige ses premières Erreurs amoureuses. G. de Sauza analyse en particulier l'influence du néoplatonisme dans le traitement de l'innamoramento, événement fondateur qui ouvre l'accès à un au-delà transcendant identifié au monde des idées platoniciennes, et dans la construction de l'image de la bien aimée, qui devient une « abstraction sans nom » dont la perfection se traduit dans l'évanescence. Ces analyses conduisent G. de Sauza à s'interroger sur l'exemplarité des relations entre le sujet lyrique et sa Dame, le recueil faisant office de parcours initiatique proposé au lecteur, mis en présence d'une conception de l'amour irréprochable. L'introduction se referme sur une brève analyse de la structure "faussement erratique » du recueil, dont l'unité se fonderait sur l'utilisation de certaines figurations du sujet lyrique au premier rang desquelles celle d'Ixion. D'où l'importance de l'image du cercle, évoquant le supplice d'Ixion, annoncée notamment par la représentation de la dame dans la gravure en forme de médaillon ouvrant le recueil et où elle tient un anneau entre ses doigts, ou suggérée par la reprise en boucle de la devise " Amour immortelle».

L'introduction est suivie de la présentation d'un protocole éditorial classique : outre les principes de transcription convenus pour rendre lisible un texte de cette époque, G. de Sauza donne une liste des erreurs manifestes qui ont été corrigées, signale des rectifications de ponctuation que la mise entre crochets rend repérables, ainsi que son choix de numéroter les poèmes par genres ( «Sonet III », «Épigramme I », etc.). Une « Bibliographie indicative », organisée en trois parties (Éditions modernes des Erreurs amoureuses, Études sur Pontus de Tyard, Études sur les Erreurs amoureuses) referme la partie introductive du volume.

Le texte des Erreurs amoureuses est édité avec soin. Il est escorté d'une annotation relativement abondante (au total 447 notes pour ce seul premier livre), en général plus étoffée que celle que l'on trouve dans les éditions de Lapp, de MacClelland ou de Kushner. Les notes sont tout aussi bien informatives ou explicatives que, souvent, interprétatives. On est particulièrement sensible au souci visible de rendre le texte compréhensible à un lectorat large, disons celui des étudiants en lettres encore peu experts dans l'étude de la poésie du XVI siècle. Les difficultés du texte, de son sens littéral, ne sont donc pas esquivées, 
et souvent G. de Sauza n'hésite pas à recourir à une très salutaire paraphrase. Peut-être ce souci le conduit-il parfois à un excès de zèle se traduisant par la présence de notes dont l'utilité n' apparaît pas clairement : par exemple, et sauf à désespérer de la perspicacité du lecteur, était-il nécessaire de préciser en note, pour le début du vers 12 du sonnet 10 (« Ha, ne laissez, ô Dieux...»), que "le sujet lyrique fait appel aux dieux païens, non au Dieu chrétien » (p. 46) ? Inversement, on apprécie particulièrement, par exemple et parmi d'autres, la très convaincante note 213 de la page 91 qui, après avoir fait le point sur les difficultés d'interprétation posées par le vers 14 du sonnet XXXIV («D’une dolente, et funebre Lidie »), identifie une source qui éclaire de façon décisive, à nos yeux, le passage. Signalons enfin une petite étourderie à corriger dans cette annotation : dans la note 416 p. 135, "Comprendre : parce qu'elle pensait ", corriger « elle» en « il».

Le texte des Erreurs amoureuses est suivi d'un glossaire référencé s'ouvrant sur une utile note signalant les formes verbales posant des difficultés d'identification et les substantifs dont le genre a changé de sens.

Cette édition est donc réalisée avec grand soin et un souci constant de rendre le texte accessible au plus grand nombre. Elle satisfait tout autant l'étudiant que le spécialiste, et son prix accessible devrait permettre au second d'initier le premier à la poésie tyardienne dans le cadre de programmes universitaires.

DANIEL MARTIn, Université d'Aix-Marseille

\section{Streete, Adrian.}

\section{Protestantism and Drama in Early Modern England.}

New York: Cambridge University Press, 2009. Pp. x, 298. ISBN 978-0-52176017-1 (hardcover) \$95.

Adrian Streete's Protestantism and Drama in Early Modern England centres its attention on how early modern subjectivity, and representations thereof in the drama of the period, are conditioned by the Protestant theologies disseminated and debated in late sixteenth-century England. Streete's book is structurally bifurcated, treating in its first half the question of how the theological positions advanced by reformers like Luther and Calvin instantiated a fundamentally 